\title{
Space-time focusing and coherence properties of supercontinua in multipass cells
}

\author{
Chao Mei $\circledast^{1, *}$ and Günter Steinmeyer $\circledast^{1,2, \dagger}$ \\ ${ }^{1}$ Max Born Institute for Nonlinear Optics and Short Pulse Spectroscopy, Max-Born-Straße 2a, 12489 Berlin, Germany \\ ${ }^{2}$ Institut für Physik, Humboldt-Universität zu Berlin, Newtonstraße 15, 12489 Berlin, Germany
}

(Received 4 January 2021; accepted 9 February 2021; published 19 March 2021)

\begin{abstract}
The situation of self-compression and concomitant supercontinuum generation in a multipass cell is analyzed in numerical simulations. This study focuses on multipass cells that contain a dielectric slab as nonlinear medium and overcompensate the dispersion of the slab with intracavity dispersive coatings. A $2 \mathrm{D}+1$ unidirectional pulse propagation equation is utilized to simulate the pulse evolution through successive passes. We observe a previously unreported effect of space-time focusing, leading to a pronounced blue shift, similar to what had been observed in filament compression experiments before. This effect competes with detrimental pulse breakup, which can nevertheless be mitigated under suitable choice of cavity parameters. We further analyze resulting coherence properties, in both the time and frequency domains. Our analysis shows highly favorable properties of multipass cell compression schemes when nonlinearity and dispersion are distributed over as many cavity passes as possible. This quasicontinuous approach is particularly promising for spectral broadening schemes that allow for stabilization of the carrier-envelope phase.
\end{abstract}

DOI: 10.1103/PhysRevResearch.3.013259

\section{INTRODUCTION}

Pulse compression is one of the key technologies for the generation of ultrashort light pulses. Compression typically relies on dispersion compensation schemes like the Treacy grating compressor [1]. By combining dispersion compensation with antecedent nonlinear spectral broadening, the pulse duration of a pulsed light source can be reduced by a substantial factor, leading to a concomitant increase of peak power. First proposed in the early 1970s, such active pulse compression reached maturity within a decade [2]. Using fiber grating compressors, compression factors of 100 and beyond $[3,4]$ were experimentally demonstrated. With the availability of shorter input pulses, a number of limiting physical mechanisms became apparent, including stimulated Raman scattering [5] and third-order dispersion [6]. As a rule of thumb, Dianov et al. suggested a minimum possible compressed pulse duration in a fiber-grating compressor of

$$
\tau_{\text {comp }}[\mathrm{ps}] \approx 0.05 \sqrt{\tau_{\text {in }}[\mathrm{ps}]},
$$

provided an input pulse duration $\tau_{\text {in }}$ [5]. This theoretical limit has been widely confirmed in subsequent experimental work. At large compression factors, another issue becomes apparent, i.e., pedestal formation. As nonlinear spectral broadening and dispersion compensation are applied in separated steps, their

\footnotetext{
*cmeibupt@gmail.com

†steinmey@mbi-berlin.de
}

Published by the American Physical Society under the terms of the Creative Commons Attribution 4.0 International license. Further distribution of this work must maintain attribution to the author(s) and the published article's title, journal citation, and DOI. effects on the phase do not ideally cancel each other out. This behavior is contrasted by adiabatic soliton compression schemes [7] and leads to the formation of satellite pulses and contrast-limiting pedestal structures [6]. While fiber-grating compressors are limited to nanojoule pulse energies, pulse compression schemes were scaled up to millijoule energies $[8,9]$ with the advent of the hollow fiber compressor $[10,11]$. By replacing the solid core of a single-mode fiber with a noble gas, these compressors could effectively eliminate issues with Raman scattering and also mitigated detrimental effects of higher order dispersion. Nevertheless, limitations by the steplike application of nonlinearity and dispersion compensation remained. Consequently, compression factors still underlie similar limitations as originally found for the fiber-grating compressor [6]. As a first step to overcome this problem, multistage hollow-fiber compressors were demonstrated with compression factors exceeding Eq. (1) by a factor of $2[12,13]$. Yet, these large compression factors were accompanied by fairly prohibitive losses, reducing peak powers rather than increasing them. Other approaches investigated self-compression in optical filaments [14,15] and offered compression of millijoule pulses in a single step. However, filament compression shows a strong radial dependence of the compression factor [16], which imposes limitations in applications. Moreover, filament compression is often accompanied by a breakup of the input pulse into two. This pulse breakup is succeeded by nonlinear diffraction of the trailing pulse due to plasma effects [17], leading to a poor overall efficiency of such schemes. Recently, nonlinear multipass cells emerged as yet another compressor geometry. These cells consist of a Herriot-like multipass cell $[18,19]$, which exploits either nonlinear broadening in a gas or a solid dielectric slab inside the cell. If used at a sufficiently large number of round trips, multipass cells enable some spatial filtering 
action if optics of tightly matched diameters are used. While this does not reach the potential of hollow-fiber compressors, multipass cells offer clearly a more favorable robustness compared to optical filaments. This acknowledged robustness has sometimes also been attributed to phase-matching issues that favor a particular Gauss-Laguerre mode [20,21]. At the same time, when cavity nonlinearity and dispersion are carefully matched, they also allow for the adiabatic pulse shaping capabilities of self-compression schemes, similar to filament compression. To this end, the dispersion compensation can be integrated into the mirror coatings of the cell. Alternatively, multipass cells can also be utilized for compression schemes with separated nonlinear and dispersive steps. Moreover, as we discuss below, multipass cells can exploit space-time focusing effects without detrimental pulse breakups and plasma-induced losses.

While many issues of the early fiber-grating compressors can be avoided with modern technology, coherence degradation imposes an ultimate barrier for any compression scheme [22]. In particular, for large spectral broadening factors and broadband spectra, input pulse energy fluctuations on the amplified spontaneous emission (ASE) level may already cause substantial pulse-to-pulse phase fluctuations at the output of the compressor [23]. As all pulse compression schemes rely on either static dispersion schemes or shapers with rather slow update times, it becomes impossible to compress all pulses in a pulse train anywhere close to their bandwidth limit. This degradation of interpulse coherence is most pronounced in photonic crystal fibers with their extremely efficient spectral broadening [24], yet is ultimately expected to appear in virtually all compression schemes conceived so far. While interpulse coherence is only relevant for applications in the time domain, a large class of applications requires spectral rather than temporal coherence of the broadened spectrum. Apart from frequency combs $[25,26]$, attosecond pulse generation schemes also heavily rely on a stable carrier-envelope phase $[27,28]$. To this end, it is important to evaluate the intrapulse coherence [29].

In the past 5 years, the idea [30] of pulse compression in multipass cells (MPCs) has become increasingly popular. MPC compression comes in two variants. At higher powers, one typically exploits the nonlinearity of gases; at lower powers, a dielectric slab is used instead, e.g., a silica plate [31,32]. Compared with hollow waveguides, MPCs are more robust and much less sensitive to pointing instabilities. More importantly, they offer additional control on pulse compression and spectral broadening through dispersion engineering and reflectivity adjustment of mirror coatings. MPCs have been widely employed to realize pulse compression at high energy. For instance, energies of 4.5 [33], 7.5 [34], 15.6 [35], and $14 \mu \mathrm{J}$ [36] have been reported experimentally. The compressed pulse energy can be significantly increased using noble gas fills. An argon-filled Herriott cell has been used for compressing $18-\mathrm{mJ}$ pulses from 1.3 ps to $41 \mathrm{fs}$ [37]. Lavenu et al. reported compression of $160-\mu \mathrm{J}, 275-$ fs input pulses to $135-\mu \mathrm{J}, 33$-fs pulses in a similar setting, yet at $150 \mathrm{kHz}$ repetition rate [38]. Pulse compression with repetition rates higher than $100 \mathrm{kHz}$ and energy larger than $1 \mathrm{~mJ}$ has been recently achieved in gas-filled MPCs [39-41]. Among these schemes, self-compression in MPCs attracted particular attention because no subsequent dispersion compensation is required postcompression. Self-compression requires net anomalous dispersion in the MPC. The latter can be most easily obtained by selecting a pump wavelength above the zero-dispersion wavelength of the dielectric medium [36]. For silica, this requires a pump wavelength above $1300 \mathrm{~nm}$. Unfortunately, wavelength scaling is not an option with media that are normally dispersive throughout like the noble gases. Another option is dispersion engineering of the mirror coating [42]. Apart from time-domain applications, another important aspect is spectral broadening [31,43]. In the most extreme demonstrations so far, a spectral supercontinuum (SC) can be generated, similar to what was reported in photonic crystal fibers [23]. While large spectral-broadening factors of 8 [31], 9 [33], and 22 [43] have been reported, octave-spanning SC and their coherence properties have not been investigated yet.

In this paper, self-compression induced octave-spanning SC generation is numerically studied in MPCs by launching high peak powers that exceed the critical power for selffocusing in silica. Going beyond previous work, we focus on the physical dynamics of self-compression and the concomitant SC generation by varying the thickness of the inserted silica plate. The spatial, temporal, and spectral evolution is investigated for multiple passes through silica plates with thicknesses of 1,5 , and $10 \mathrm{~mm}$. The resulting coherence properties are analyzed, in particular with a view on the intrapulse coherence of the generated SC. In order to elucidate possible consequences for carrier-envelope phase stabilization [27], we investigate phase fluctuations between a fundamental frequency component and its second harmonic. To this end, we further analyze the influence of the input pulse contrast, as this may vary widely between different laser systems.

\section{DESIGN OF STATIONARY MULTIPASS CELL}

The concept of MPCs dates back to the emergence of resonators with spherical or aspherical mirrors in early 1960s. Such cells were originally designed for interferometers [18] and optical delay lines [19]. MPCs can be regarded as similar to laser cavities that enforce or restore an eigenmode of the cavity if the input beam is not properly mode matched. This spatial filtering action is beneficial also for their use as compressor as it avoids beam breakup or the spatial inhomogeneities of filament compression. The eigenmode can be calculated by the ABCD matrix formalism, which is determined by the radius of curvature of mirror $R$ and distance of $L$ between two cavity mirrors. When $L<R$, the waist radius $w_{0}$ at the center of the MPC and the beam radius on the mirrors $w_{m}$ are both small. In terms of nonlinear phase accumulation, this kind of cavity is most suitable because of the resulting high nonlinear coefficient and the unconditionally stable beam size. However, the short length of this kind of cavity renders input and output coupling difficult. The limiting case $L=R$ is also known as confocal configuration. Now $w_{0}$ is much larger than in the case of $L<R$, i.e., a higher input power is needed for obtaining identical nonlinear phase accumulation. In addition, input and output coupling become virtually impossible as the beam reaches the identical position on the mirror after only two round trips, forming a two-spot pattern on each mirror. While a recent report showed 

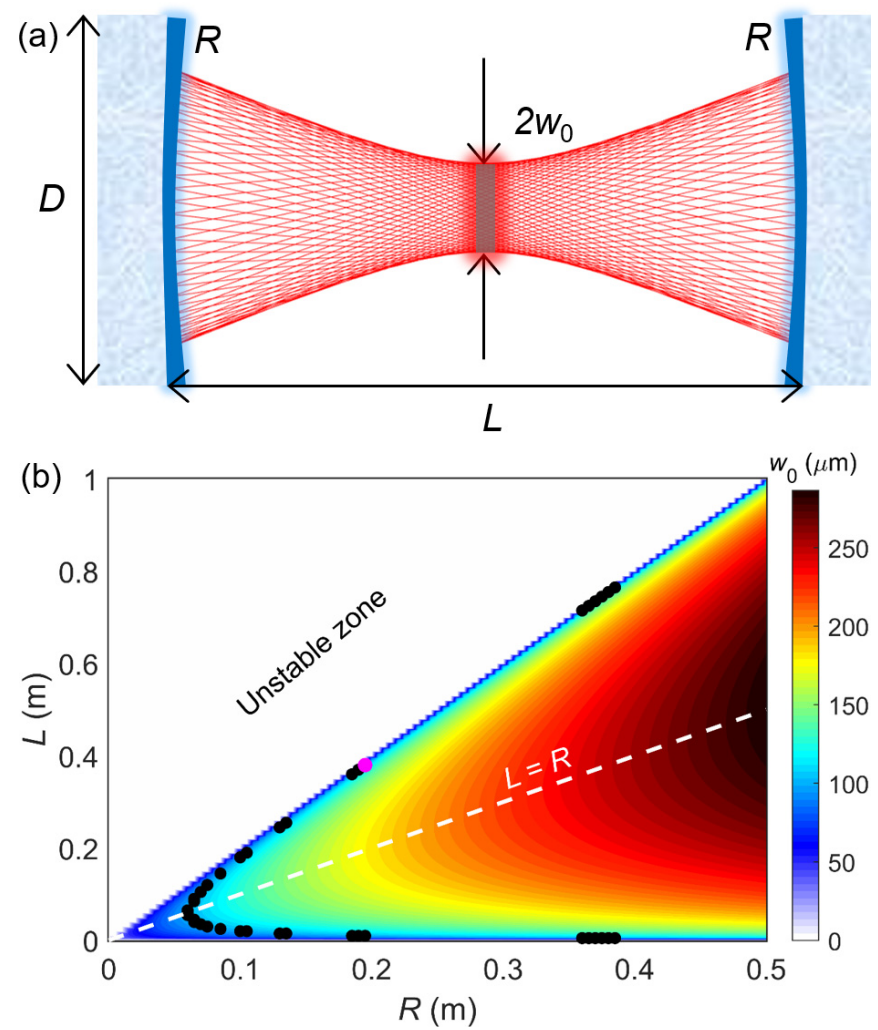

FIG. 1. (a) 2D sketch of investigated MPC. (b) Distribution of beam waist $w_{0}$ on an $L-R$ map, $L=R$ (white dashed line), $w_{0}=$ $100 \mu \mathrm{m}$ (black dots), and best performance parameters chosen for the simulation $R=195 \mathrm{~mm}, L=380 \mathrm{~nm}$ (pink dot).

that nonlinear pulse compression can be achieved in a gasfilled confocal MPC, the constructed $4 f$ imaging structure is rather sophisticated, incorporating four concave mirrors [39]. Finally, when $R<L<2 R, w_{0}$ is decreasing with increasing $L$ at fixed $R$. At the same time $\left(w_{0} / w_{m}\right)^{2}=2 R /(2 R-L)$, which means the cavity length approaches $2 R$, resulting in a infinitely large value of $w_{m}$. This condition could be helpful to increase the damage threshold of the cavity mirrors. When $L=2 R$, the beam traveling in the cavity tends to be reimaged onto itself in successive round trips. This exact situation should be avoided in self-compression and SC generation because of resulting poor spatial homogenizing. In experimental implementations, $L$ should therefore be slightly shorter than $2 R$, e.g., as demonstrated in Refs. [34] and [43].

The conceptual setup of the MPC assumed in the following simulations is shown in Fig. 1(a). The cell consists of two concave mirrors with diameter $D=50.8 \mathrm{~mm}$ and radius of curvature $R$. The concave surface oriented toward the center of MPC is coated by a highly reflective (HR) mirror structure with anomalous group delay dispersion (GDD). The value of the anomalous GDD is optimized to overcompensate for the normal GDD of the silica plate, resulting in a net anomalous GDD for self-compression. The GDD values of silica plates and HR coatings are listed in Table I. In the following simulation, we assume perfect components, i.e., highly reflecting mirrors with $100 \%$ reflectivity and smooth dispersion properties without dispersion oscillations [44]. Moreover, we neglect
TABLE I. GDD $\left(\mathrm{fs}^{2}\right)$ of optical elements in MPC.

\begin{tabular}{lccc}
\hline \hline Thickness $(\mathrm{mm})$ & Silica plate & Mirror coating & Net value \\
\hline$d=10$ & 179 & -180 & -1 \\
$d=5$ & 89.5 & -120 & -30.5 \\
$d=1$ & 17.9 & -25 & -7.1 \\
\hline \hline
\end{tabular}

Fresnel losses at the interfaces of the nonlinear medium. Assuming standard broadband AR coatings, one can estimate typical Fresnel losses of $<20 \%$ for the maximum number of 34 passes and a comparable number for reflection losses. We therefore conclude that accounting for these losses does not lead to any qualitative change in the results discussed below. In a similar fashion, we also neglected dispersive and nonlinear effects of the ambient medium. Even for the thinnest 1-mm silica plates, air nonlinearity and dispersion are expected to be an order of magnitude below the respective silica values. Moreover, if such effects are found to be detrimental, this problem can either be mitigated by replacing intracavity air by helium or evacuating the entire cavity [45].

By changing the launched beam angle and its position on the concave-convex mirror, different numbers of passes and varying beam waist $w_{0}$ can be obtained accordingly. For the 10-, 5-, and 1-mm silica plates, the corresponding numbers of passes are 6,11 , and 34 , respectively. The value of $w_{0}$ is determined by parameters $R$ and $L$. The distribution of $w_{0}$ on the $L-R$ map is calculated by ABCD matrix formalism, as shown in Fig. 1(b). Within the ranges of vertical and horizontal coordinates, the area covered area by $L<2 R$ exhibits a symmetrical distribution along $L=R$. In the regime of $L>2 R$, the cavity becomes unstable, i.e., this situation will be avoided. Therefore, the designed MPC should be slightly detuned from the stability limit of $L=2 R$. Here the value of $w_{0}$ is chosen as $100 \mu \mathrm{m}$. To find the specific combinations of $L$ and $R$ that satisfy $w_{0}=100 \mu \mathrm{m}$, we marked all values of $100-\mu \mathrm{m}$ with black solid dots on the $L-R$ map. We selected a value of $L=380 \mathrm{~mm}$ and a corresponding $R=195 \mathrm{~mm}$, which satisfies the stationary condition of $L<2 R$. The operation point is highlighted on the $L-R$ map as pink solid dot in Fig. 1(b). A recent theoretical calculation predicted that strong ionization effects may be triggered for gas-filled MPCs [46] in close proximity to the stability limit. In this work, the influence of ionization in silica plates will be discussed in Sec. IV.

\section{PROPAGATION MODEL}

In our propagation model, we neglect nonlinear optical processes outside the silica slab. In particular, we also neglect nonlinear effects inside the mirror coatings. To numerically describe the space-time evolution in the silica plate, the $2 \mathrm{D}+1$ unidirectional pulse propagation equation (UPPE) is utilized. UPPE was first established by Kolesik et al. [47] to describe the space-time propagation of few-cycle pulse. This method was widely adapted by others [48-50]. Compared to the nonlinear Schrödinger equation (NLSE), the UPPE can provide a more accurate description of pulse propagation, in particular, when it comes to extremely short or broadband 
electromagnetic pulses. Moreover, we restrict ourselves to describing the $z$ propagation with the UPPE as the transverse size is sufficiently large compared to the wavelength, i.e., the corresponding paraxial approximation holds. Mathematically, $z$ propagation is modeled by the UPPE in cylindrical coordinates as [51]

$$
\begin{aligned}
\partial_{z} \widetilde{\mathcal{E}}_{k_{\perp}}(\omega, z) & =i k_{z} \widetilde{\mathcal{E}}_{k_{\perp}}(\omega, z)+\frac{i \omega^{2}}{2 \epsilon_{0} c^{2} k_{z}} \widetilde{\mathcal{P}}_{\perp}(\omega, z) \\
& -\frac{\omega}{2 \epsilon_{0} c^{2} k_{z}} \widetilde{\mathcal{J}}_{\perp}(\omega, z),
\end{aligned}
$$

where $k_{z}=\sqrt{k^{2}(\omega)-k_{\perp}^{2}}$ is the $z$ component of the wave vector with modulus $k(\omega)=n(\omega) \omega / c$. Here $c$ is the light speed in vacuum and $\epsilon_{0}$ is the permittivity of free space. The UPPE is written in a spectral representation, i.e., it describes the evolution of the spectrum rather than that of a pulse. Unlike the partial differential form of the NLSE, the UPPE is a system of ordinary differential equations for the complex spectral field $\widetilde{\mathcal{E}}_{k_{\perp}}(\omega, z)$. However, only the spectral amplitude $\widetilde{A}$, which is related to $\widetilde{\mathcal{E}}$ via $\widetilde{\mathcal{E}}_{k_{\perp}}(\omega, z)=\widetilde{A}_{k_{\perp}}(\omega, z)\left(i k_{z} z\right)$, enters into the nonlinearity [51]

$$
\partial_{z} \widetilde{A}_{k_{\perp}}(\omega, z)=\frac{i \omega^{2}}{2 \epsilon_{0} c^{2} k_{z}} \widetilde{\mathcal{P}}_{\perp}(\omega, z)-\frac{\omega}{2 \epsilon_{0} c^{2} k_{z}} \widetilde{\mathcal{J}}_{\perp}(\omega, z) .
$$

In Eq. (2), $\widetilde{\mathcal{P}}_{\perp}(\omega, z)$ is a nonlinear polarization term, including the instantaneous Kerr effect and the noninstantaneous Raman effect. This term can be rewritten in the time domain [51]

$$
\mathcal{P}_{\perp}(t)=2 \epsilon_{0} n_{0} n_{2}\left[\left(1-f_{R}\right) I(t)+f_{R} \int_{0}^{\infty} \mathcal{R}(\tau) I(t-\tau) d \tau\right],
$$

where $n_{0}$ denotes the linear refractive index at pump center frequency $\omega_{0}, n_{2}$ is the nonlinear refractive index whose frequency dependence is neglected, $f_{R}$ stands for the fractional contribution of the Raman response, and $\mathcal{R}(t)$ represents a memory function that describes the Raman effect. Finally, $I(t)=2|\mathcal{E}|^{2} /\left(\pi w_{0}^{2}\right)$ is the light intensity [52].

The nonlinear absorption is described by a plasma-induced current $\mathcal{J}$, which accounts for the average dissipated power caused by optical ionization. The time-dependent evolution equation for the plasma current density is [51]

$$
\frac{\partial \mathcal{J}}{\partial t}+\frac{\mathcal{J}}{\tau_{\mathrm{c}}}=\frac{q_{\mathrm{e}}^{2}}{m_{\mathrm{e}}} \rho \mathcal{E}
$$

where $\rho$ denotes the electron density and $\tau_{\mathrm{c}}$ stands for the average collision time of the electron, $q_{\mathrm{e}}$ is the elementary charge, and $m_{\mathrm{e}}$ is the reduced electron-hole mass. The real part of $\mathcal{J}$ accounts for plasma absorption, and the imaginary part represents plasma defocusing. The time-domain evolution of $\rho(r, t)$ in Eq. (5) is governed by a rate equation [51]:

$$
\frac{\partial \rho}{\partial t}=W_{\text {ofi }}(I)\left(\rho_{\mathrm{nt}}-\rho\right)+W_{\mathrm{ava}}(I) \rho .
$$

Here $W_{\text {ofi }}=\sigma_{\mathrm{K}} I^{K}$ denotes the optical field ionization in multiphoton regime, $K=8$ is photon number defined by $\bmod \left[U_{\mathrm{i}} /\left(\hbar \omega_{0}\right)\right]+1, \hbar$ is the reduced Planck constant, $\rho_{\mathrm{nt}}$ represents the density of neutral atoms, $W_{\mathrm{ava}}=\sigma\left(\omega_{0}\right) I / U_{\mathrm{i}}$ represents the rate of avalanche ionization proportional to the pulse intensity, $U_{\mathrm{i}}$ is the band gap of silica, and $\sigma_{\mathrm{K}}$ represents
TABLE II. Simulation parameters used for solving UPPE

\begin{tabular}{lccccc}
\hline \hline & \multicolumn{2}{c}{ Pulse parameters } & \multicolumn{2}{c}{ Material parameters } & \\
Symbol & Value & Unit & Symbol & Value & Unit \\
\cline { 4 - 6 } & 1.03 & $\mu \mathrm{m}$ & $n_{2}$ & $2.2 \times 10^{-20}$ & $\mathrm{~m}^{2} / \mathrm{W}$ \\
$\lambda_{0}$ & 17 & $\mathrm{MW}$ & $U_{\mathrm{i}}$ & 9 & $\mathrm{eV}$ \\
$P_{\text {in }}$ & 100 & $\mu \mathrm{m}$ & $m_{\mathrm{e}}$ & $7.8 \times 10^{-31}$ & $\mathrm{~kg}$ \\
$w_{0}$ & 177 & $\mathrm{GW} / \mathrm{cm}^{2}$ & $\tau_{\mathrm{c}}$ & 1 & $\mathrm{fs}$ \\
$I_{\text {in }}$ & 100 & $\mathrm{fs}$ & $\rho_{\mathrm{nt}}$ & $2.1 \times 10^{28}$ & $\mathrm{~m}^{-3}$ \\
$t_{\mathrm{F}}$ & 1.8 & $\mu \mathrm{J}$ & $n_{0}$ & 1.45 & \\
$E_{\text {in }}$ & 10 & $\mathrm{~cm}$ & $f_{\mathrm{R}}$ & 0.18 & \\
$f$ & & & & & \\
\hline \hline
\end{tabular}

${ }^{\mathrm{a}} n_{2}$ is cited from Ref. [58] and others are cited from Ref. [49].

the cross section of multiphoton ionization

$$
\begin{aligned}
\sigma_{K} \simeq & \frac{2 \omega_{0}}{9 \pi}\left(\frac{m_{\mathrm{e}} \omega_{0}}{\hbar}\right)^{\frac{3}{2}} \Phi\left(\sqrt{2\left[K-U_{i} /\left(\hbar \omega_{0}\right)\right]}\right) e^{2 K} \\
& \times\left(\frac{q_{e}^{2}}{16 m_{e} \omega_{0} U_{i}}\right)^{K},
\end{aligned}
$$

where $\Phi(x)=e^{-x^{2}} \int_{0}^{x} e^{y^{2}} \mathrm{~d} y$. Equation (7) is a simplified version of Keldysh theory [53], from which we estimate $\sigma_{\mathrm{K}}=3.72 \times 10^{-97} \mathrm{~s}^{-1} \mathrm{~cm}^{16} / \mathrm{W}^{8} . \sigma\left(\omega_{0}\right)$ is the inverse bremsstrahlung coefficient [51]

$$
\sigma\left(\omega_{0}\right)=\frac{\omega_{0}^{2} \tau_{\mathrm{c}}}{n\left(\omega_{0}\right) c \rho_{\mathrm{c}}} \frac{1}{1+\omega_{0}^{2} \tau_{\mathrm{c}}^{2}} .
$$

Here $\rho_{\mathrm{c}}=\epsilon_{0} m_{\mathrm{e}} \omega_{0}^{2} / q_{\mathrm{e}}^{2}$ is the critical plasma density. It should further be noted that we neglect the electron recombination in Eq. (6) because input pulse width and recombination time constant (150 fs) [54] are comparable; cf. Ref. [49].

The input pulse is assumed to be Gaussian

$$
\mathcal{E}(r, t, z=0)=\sqrt{I_{\text {in }}} \exp \left[-\frac{r^{2}}{w_{0}^{2}}-\frac{t^{2}}{t_{0}^{2}}-i k_{0} \frac{r^{2}}{2 f}\right],
$$

where $I_{\text {in }}$ is the input light intensity calculated as 177 $\mathrm{GW} / \mathrm{cm}^{2}$. Such an intensity is far below the ionization threshold of $10-20 \mathrm{TW} / \mathrm{cm}^{2}$ [55]. $P_{\text {in }}$ is the input peak power, and $t_{0}$ and $f$ denote the pulse width and effective focal length, respectively. $k_{0}=2 \pi / \lambda_{0}$ is the wave vector at pump center wavelength. In the following, we always refer to pulse widths at the full-width at half-maximum (FWHM), $t_{\mathrm{F}}$. For Gaussian pulses, the conversion relationship between $t_{0}$ and $t_{\mathrm{F}}$ is $t_{\mathrm{F}}=$ $\sqrt{2 \ln 2} t_{0}$, leading to an input energy $E_{\text {in }}=\sqrt{\pi /(4 \ln 2)} t_{\mathrm{F}} P_{\text {in }}$. The initial values of $t_{\mathrm{F}}$ and $w_{0}$ are assumed as $100 \mathrm{fs}$ and $100 \mu \mathrm{m}$, respectively. The initial peak power $P_{\text {in }}=3.2 P_{\mathrm{c}}$, where $P_{\mathrm{c}}$ is the critical power for self-focusing given by [56]

$$
P_{\mathrm{c}} \approx \lambda_{0}^{2} /\left(2 \pi n_{0} n_{2}\right) .
$$

The values of $P_{\mathrm{c}}$ and $P_{\text {in }}$ are calculated as 5.3 and $17 \mathrm{MW}$, respectively, and the input energy is $1.8 \mu \mathrm{J}$. Other simulation parameters are summarized in Table II. In terms of numerical implementation, Eq. (2) can be transformed from frequency domain to space-time domain, namely, $k_{\perp} \rightarrow r$ and $\omega \rightarrow t$, so that Eqs. (4)-(6) can be coupled with Eq. (2). This process is accomplished by the Hankel transformation in the radial domain [57] and an inverse Fourier transformation in the frequency domain at every $z$ step. The size of the $z$ step is 

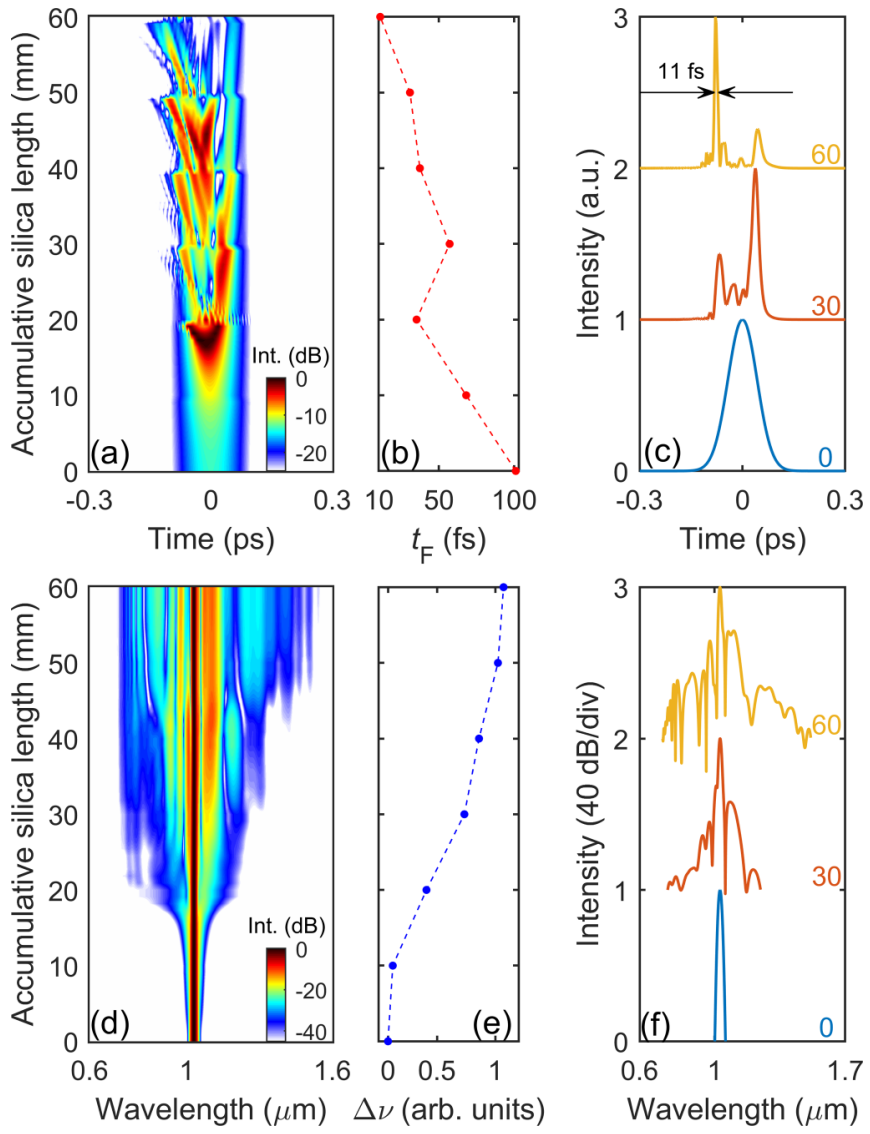

FIG. 2. The 10-mm case: (a) Temporal evolution and (b) evolution of pulse duration $t_{\mathrm{F}}$ vs accumulative propagation length in silica. (c) Temporal waveforms at the input (blue) and after $30 \mathrm{~mm}$ (red) and $60 \mathrm{~mm}$ (yellow) of propagation. (d) Spectral evolution and (e) evolution of the spectral width in octave units. (f) Spectral waveforms at the input (blue) and after 30 (red) and $60 \mathrm{~mm}$ (yellow) of propagation.

automatically adapted by using the ODE45 algorithm that is built in the software of MATLAB to save computational time. Simulation results will be discussed in the following section.

\section{ANALYSIS OF TEMPORAL, SPECTRAL, AND SPATIAL CHARACTERISTICS}

We first consider the case of a 10 -mm-thick silica slab, as shown in Fig. 2. The input beam was assumed to experience six passes in the MPC, corresponding to a total cumulated length of $60 \mathrm{~mm}$ in the nonlinear medium. Figure 2(a) depicts the evolution of temporal pulse profile at $r=0$. At $18 \mathrm{~mm}$ of accumulated propagation distance, the spectrum starts to rapidly broaden. This finding agrees with the predicted 17.5$\mathrm{mm}$ value for the self-focusing distance in the Marburger equation [56]

$$
z_{s}=\frac{0.367 z_{\mathrm{R}}}{\sqrt{\left[\left(P_{\mathrm{in}} / P_{c}\right)^{1 / 2}-0.852\right]^{2}-0.0219}},
$$

where $z_{\mathrm{R}}$ is the Rayleigh range given by $z_{\mathrm{R}}=\pi n_{0} w_{0}^{2} / \lambda$. After initial self-focusing, a collapse of the temporal profile occurs, resulting in a pulse split, which appears to impose a critical threshold that must not be exceeded for meaningful application of the compression scheme. However, similar to what was observed in filamentation [16,17], the trailing pulse subsequently dies out, and the leading satellite pulse is compressed to 11 fs, i.e., a ninefold self-compression. In stark contrast to filament self-compression, though, additional calculations show that the main peak of the compressed pulse contains $0.97 \mu \mathrm{J}$ energy, corresponding to a compression efficiency of $54 \%$.

Figure 2(d) depicts the evolution of spectra at $r=0$ as a function of the accumulated dispersive and nonlinear effects in silica. The spectra appear gradually broadened due to various nonlinear effects including self-phase modulation, self-steepening, and the Raman effect. The spectral width is measured in octaves, as defined by $\Delta v=\log _{2}\left(\lambda_{\max } / \lambda_{\min }\right)$, where $\lambda_{\max }$ and $\lambda_{\min }$ are the longest and shortest wavelengths at a given level, respectively. In experimental work on supercontinua, one typically uses a $-40 \mathrm{~dB}$ level [59], dictated by the dynamic range of optical spectrum analyzers. The evolution of the spectral width in Fig. 2(e) is roughly inversely correlated with the evolution of the pulse width in Fig. 2(f) and exceeds the octave after $5 \mathrm{~cm}$ of accumulated propagation in silica. The resulting $\lambda_{\max }$ and $\lambda_{\min }$ after $5 \mathrm{~cm}$ of propagation are 1.52 and $0.72 \mu \mathrm{m}$, respectively, corresponding to an eight hundred nanometer bandwidth. Considering the initial spectral width of $57 \mathrm{~nm}$, we observe spectral broadening by a factor 14. Nevertheless, the influence of plasma induced by optical ionization is found negligible; i.e., the largest electron density $\rho$ at the self-focusing distance is estimated as $10^{12} \mathrm{~cm}^{-3}$. This value is substantially lower than the required electron density $\left(>10^{18} \mathrm{~cm}^{-3}\right)$ for a plasma-induced pulse collapse [60].

To improve the compression factor, the output position needs to be carefully chosen. To this end, a thinner silica plate with $d=5 \mathrm{~mm}$ is considered in the following. The GDD of the mirror coating is decreased to $-120 \mathrm{fs}^{2}$ per pass accordingly. The number of total passes is increased to 11 in order to accumulate sufficient nonlinear phase. Simulation results for this case are shown in Fig. 3, again both in the time and frequency domains. Temporal self-focusing still kicks in at $18 \mathrm{~mm}$, as shown in Fig. 3(a). Subsequently, one can see a similar scenario as for the 5-mm case [3(c)]. The pulse splits into two, with an initially stronger trailing satellite, which eventually disappears and gives way to a compressed leading one with a duration of only $6.6 \mathrm{fs}$, corresponding to only 1.9 optical cycles. The energy contained in the latter is calculated as $0.5 \mu \mathrm{J}$, indicating a compression efficiency of $28 \%$, which is again much higher than typically observed in filament compression. The concomitant spectral evolution is shown in Fig. 3(d). While the final compressed pulse duration is shorter in the 5-mm case, the octave coverage appears slightly reduced; compare Figs. 3(e) and 2(e). We further noticed that the spectra of the 5-mm case appear to be blue shifted with respect to the $10-\mathrm{mm}$ case, which is indicative of a stronger influence of self-steepening. In filamentation, one often suspects plasma effects behind this blue shift [61]. However, the maximum electron density at the self-focusing distance is now calculated as $3.5 \times 10^{7} \mathrm{~cm}^{-3}$, i.e., 11 orders of magnitude smaller than electron density required for sensible plasmabased self-steepening action here. We therefore conclude that 

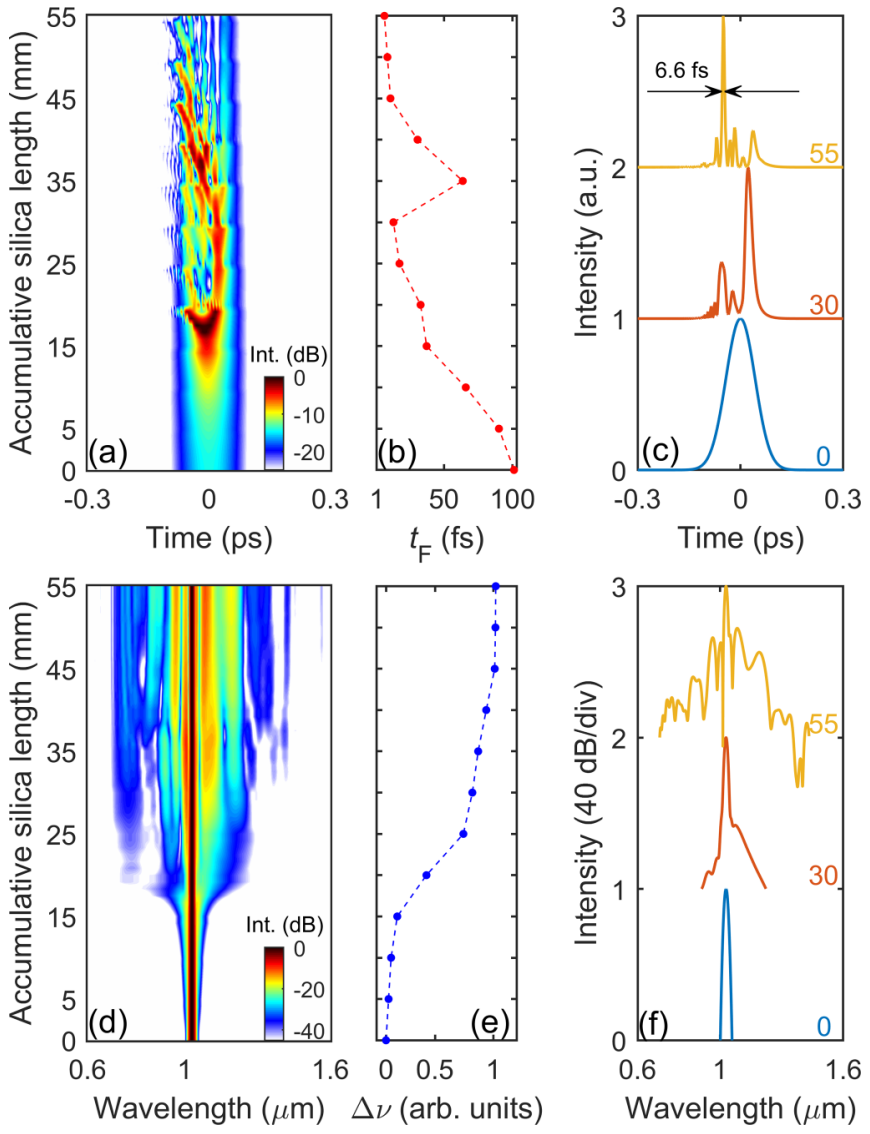

FIG. 3. The 5-mm case: Otherwise identical to Fig. 2. The yellow trace in panel (f) refers to an accumulated propagation length of only 55 rather than $60 \mathrm{~mm}$.

it is in fact space-time focusing [62] that causes the blue shift here. This effect can be understood as a self-steepening effect of the diffraction term in the $2 \mathrm{D}+1$ propagation model and mostly depends on pulse shortness rather than intensity. The space-time focusing effect must not be confused with detrimental space-time coupling effects [38].

In summary, the thinner silica plate effectively leads to a reduced accumulation of the nonlinear optical phase. However, space-time focusing still renders the SC octave spanning. In order to further analyze this trend, we therefore switch to the quasicontinuous case of $d=1 \mathrm{~mm}$; i.e., we now require a total of several ten cavity round trips for sufficient spectral broadening. Simulation results are shown in Fig. 4. As in the former two cases, self-focusing still takes place at approximately $18 \mathrm{~mm}$. Compared to the previous two cases, however, we do not see a catastrophic pulse collapse anymore. Instead, we observe a rather smooth self-compression process, leading only to a faint trailing satellite in the time domain. The pulse duration $t_{\mathrm{F}}$ in Fig. 4(b) steadily decreases until about $25 \mathrm{~mm}$. Subsequent variation of $t_{\mathrm{F}}$ is comparatively gentle, which means that no pronounced pulse splitting occurs. The input 100 -fs pulse is eventually compressed to $6.6 \mathrm{fs}$ with $0.6-\mu \mathrm{J}$ energy contained in the main peak of the compressed pulse, as shown in Fig. 4(c). The calculated compression efficiency is $34 \%$. The spectral evolution along the accumulative silica length is shown in Fig. 4(d). Significant spectral
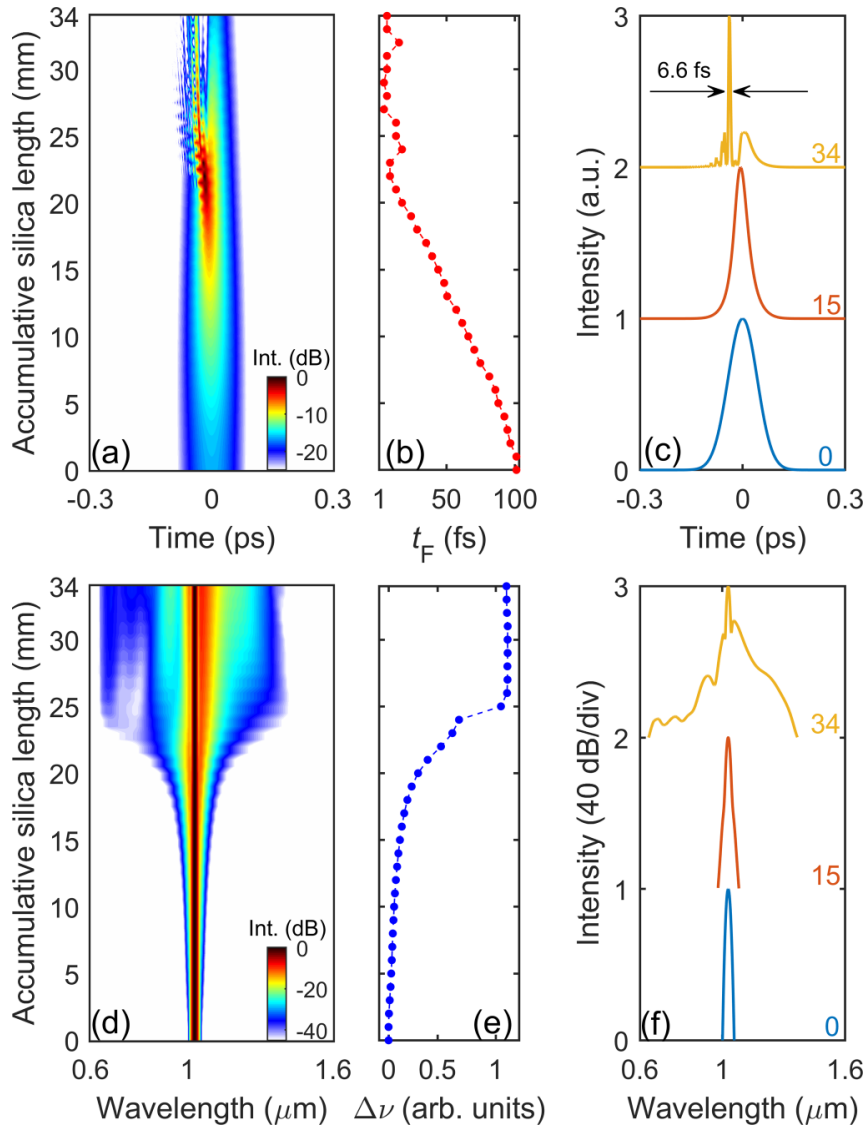

FIG. 4. The 1-mm case: Identical to Fig. 2, but for the yellow and red traces in panel (f), which refer to accumulated propagation lengths of only 15 and $34 \mathrm{~mm}$, rather than 30 and $60 \mathrm{~mm}$, respectively.

broadening is observed until the accumulative silica length reaches $25 \mathrm{~mm}$. After that, the spectral width remains almost unchanged because the nonlinear phase accumulation after the self-focusing is negligible. This is further verified in Fig. 4(e); i.e., after $25 \mathrm{~mm}$ of propagation, spectral coverage remains almost constant. Moreover, the cutoff wavelength now further lowered to $641 \mathrm{~nm}$, which is more than $60 \mathrm{~nm}$ smaller than previously observed [Fig. 4(f)], i.e., the space-time focusing action further increased. The calculated factor of spectral broadening is 12.7 , and the maximum electron density at the self-focusing distance is now $5 \times 10^{6} \mathrm{~cm}^{-3}$. This value is the smallest among all three cases considered. These simulation results further consolidate the previously observed trend of thinner silica plates leading to a stronger blue shift.

The evolution of the radial field at $t=0$ is shown in Fig. 5 . Similar to the onset of spectral broadening, the self-focusing induced spatial compression happens around $18 \mathrm{~mm}$. The initial beam size reduces from 100 to about $20 \mu \mathrm{m}$, as marked by a black solid circle in Fig. 5(a). After the nonlinear focus, some part of the beam is diffracted off in several steps. The first nonlinear focus results in the initial beam splitting; i.e., the central part of the temporal beam profile is diffracted off. In the subsequent steps, the trailing pulse accumulates losses, which eventually cause this pulse to die out. These nonlinear diffraction losses [17] therefore explain the vanishing of the 

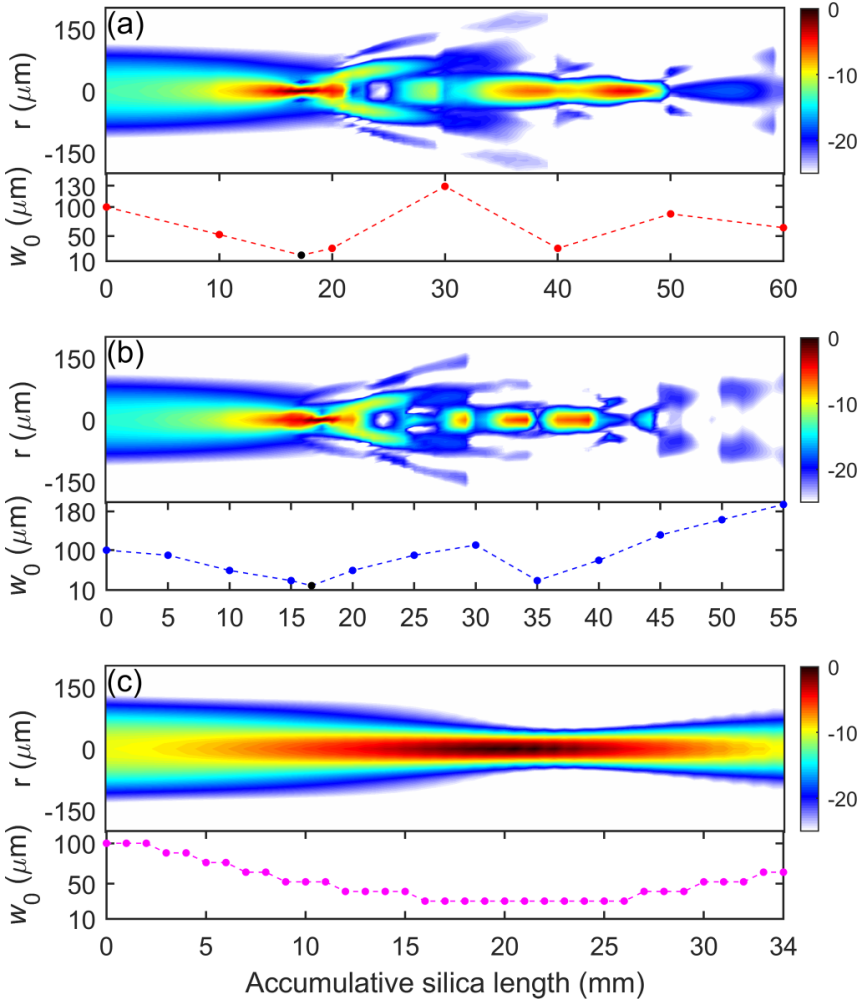

FIG. 5. Upper panel: evolution of radial field. Lower panel: evolution of $w_{0}$ vs propagation length length for (a) $=d=10 \mathrm{~mm}$, (b) $d=5 \mathrm{~mm}$, and (c) $d=1 \mathrm{~mm}$.

trailing satellite in Figs. 2(b) and 3(b). Moreover, assuming an initially symmetric split of energy into three pulses, overall compression efficiencies near 33\% appear plausible. The beam size $w_{0}$ in Figs. 5(a) and 5(b) undergoes two refocusing cycles, with minima at 18 and near $40 \mathrm{~mm}$. The primary focus coincides with the onset of rapid spectral broadening and pulse splitting, whereas the secondary refocusing appears to be correlated with the vanishing of the trailing satellite. We compute a resulting fluence of $1.8 \mu \mathrm{J} /\left[\pi \times(19.3 \mu \mathrm{m})^{2}\right] \approx$ $0.15 \mathrm{~J} / \mathrm{cm}^{2}$, which is well below typical damage thresholds of $\approx 1 \mathrm{~J} / \mathrm{cm}^{2}$ [39]. While the scenario is qualitatively very similar for the 10- and 5-mm cases, a completely different quasicontinuous behavior emerges for the 1-mm case; see Fig. 5(c). Now the evolution is comparatively smooth, leading to a focusing event at $23 \mathrm{~mm}$, with a single minimum $w_{0}=30 \mu \mathrm{m}$; i.e., significantly larger than in the previously discussed cases. This nonlinear focus event is not accompanied by any beam collapse or apparent defocusing of parts of the temporal profile.

The space-time characteristics are further investigated in Fig. 6. The input space-time map is assumed to be Gaussian input in space and time; see Fig. 6(a). For the 10-mm case in Fig. 6(b), pulse splitting leads to the appearance of multiple peaks. Both the main and secondary peaks are located on axis at $r=0$, yet at different temporal positions $(t=-80$ and $45 \mathrm{fs}$ ). Off-center components have been stripped off in the nonlinear focus events. In Fig. 6(b), these off-center components form two pronounced rings near zero delay and at $t=30 \mathrm{fs}$. In these rings, the intensity maximum appears off
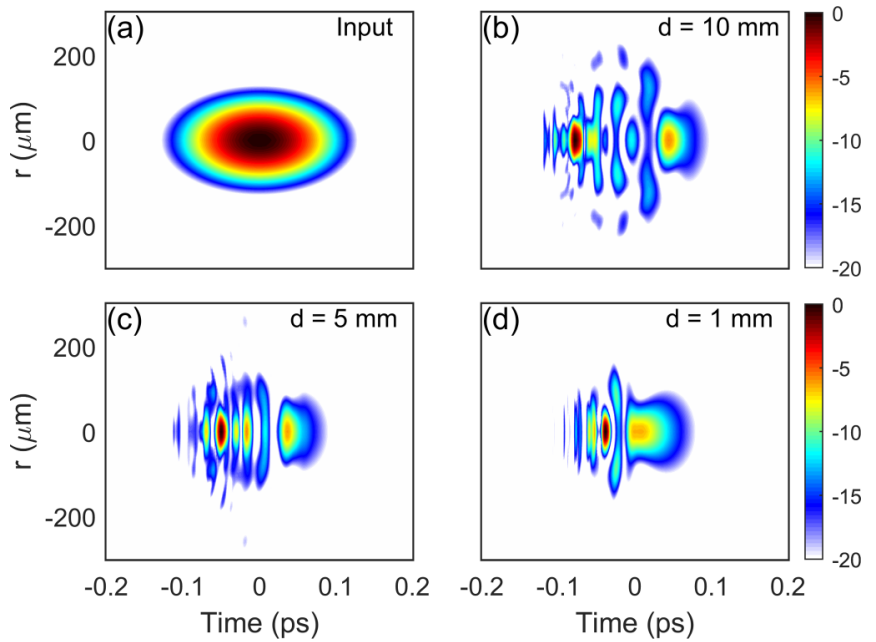

FIG. 6. Space-time map for (a) input pulse and (b) output pulse for $=d=10 \mathrm{~mm}$, (c) $d=5 \mathrm{~mm}$, and (d) $d=1 \mathrm{~mm}$.

center near $r=100 \mu \mathrm{m}$. The formation of such rings becomes less pronounced in the 5-mm case, cf. Fig. 6(c), and virtually disappears in the 1-mm case of Fig. 6(d), while spatiotemporal effects become much less pronounced in the quasicontinuous case. It needs to be emphasized that spatiotemporal effects in Fig. 6 remain rather benign. Most of the energy contents remains in the fundamental Laguerre-Gauss mode $\mathrm{LG}_{00}$, and only a small fraction is transferred into the next higher $\mathrm{LG}_{10}$ mode by self-focusing. The latter mode experiences additional diffractive losses due to limited coating diameters. This effect is difficult to simulate within a $2 \mathrm{D}+1$ code without making explicit assumptions. Moreover, $\mathrm{LG}_{10}$ content can be stripped off by external mode filtering, if required. Consequently, it appears rather straightforward to maintain a high spatiotemporal coherence, exploiting some additional spatial filtering either within the multipass cell or external to it.

The nonlinear phase accumulation during the spectral broadening is measured by the $B$ integral. Here we consider both the local and the accumulative $B$ integral in order to clearly see the phase variation over many passes. The local $B$ integral is calculated over a small number of $z_{\mathrm{s}}$ steps,

$$
B_{\text {int }}=\frac{2 \pi n_{2}}{\lambda} \int_{0}^{N_{\mathrm{s}} z_{\mathrm{s}}} \frac{P_{\text {peak }}(z)}{A_{\mathrm{eff}}(z)} d z,
$$

where $N_{\mathrm{s}}$ is the step number, $P_{\text {peak }}(z)$ and $A_{\text {eff }}(z)$ are local peak power and effective mode area, respectively, and $A_{\text {eff }}(z)=$ $\pi w_{0}^{2}(z)$ is calculated from the local beam size. $z_{\mathrm{s}}$ is chosen as $1 \mathrm{~mm}$. The selected $z_{\mathrm{s}}$ provides a specific $N_{\mathrm{s}}$ of 66,55 , and 34 for the 10-, 5-, and 1-mm cases, respectively. In contrast, the accumulative $B$ integral is the superposition of local $B$ integrals at every step. The calculation results are shown in Fig. 7. The accumulative $B$ integral increases in a stepwise fashion at lower values of $d$, and one can see in the local $B$ integral that this accumulation occurs in a rather irregular fashion mostly at the two refocusing events; see Figs. 7(a) and 7(b). In contrast, for the 1-mm case, the nonlinear phase is accumulated within about $10 \mathrm{~mm}$ in a single focusing event; see Fig. 7(c). 

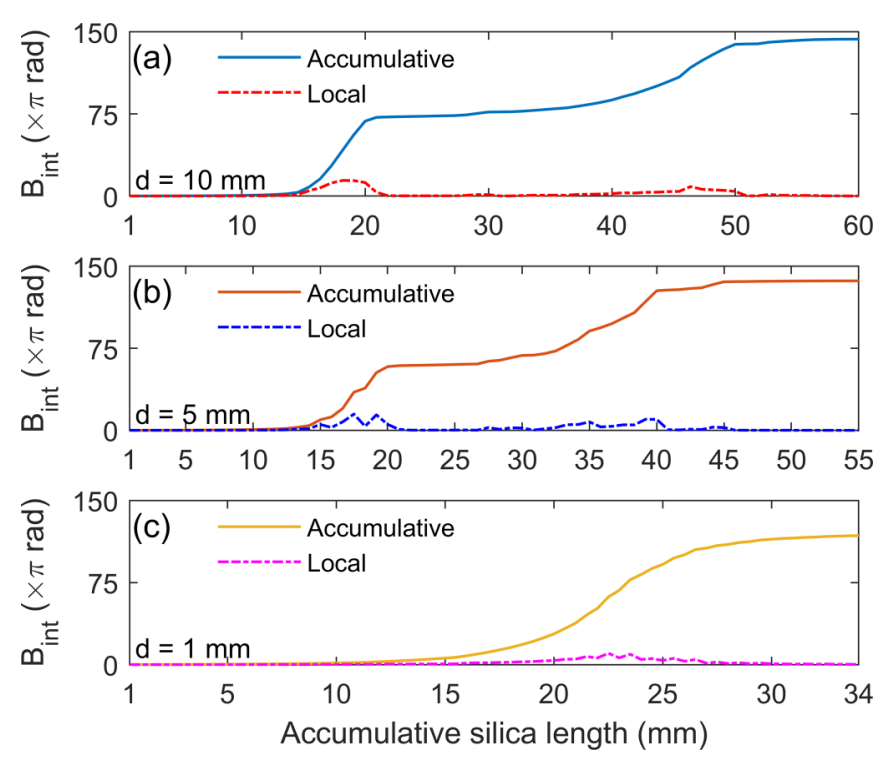

FIG. 7. Local (dashed dot curves) and accumulative (solid curves) $B$ integrals for the (a) $10-\mathrm{mm}$ case, (b) 5-mm case, and (c) 1 -mm case.

\section{COHERENCE ANALYSIS}

One important aspect for applications of a SC is its coherence properties. Coherence is traditionally defined in the time domain; i.e., in the case of an ultrashort pulse train, one typically refers to the coherence within an ensemble of temporal envelopes [63]. This kind of coherence is referred to as pulse-to-pulse or interpulse coherence and is relevant for temporal compression of individual pulses within the train close to their respective Fourier limit,

$$
\left|g_{12}^{(1)}\left(\lambda, t_{1}-t_{2}\right)\right|=\left|\frac{\left\langle\mathcal{E}_{i}^{*}\left(\lambda, t_{1}\right) \mathcal{E}_{j}\left(\lambda, t_{2}\right)\right\rangle_{i \neq j}}{\left[\left\langle\left|\mathcal{E}_{i}\left(\lambda, t_{1}\right)\right|^{2}\right\rangle\left\langle\left|\mathcal{E}_{j}\left(\lambda, t_{2}\right)\right|^{2}\right\rangle\right]^{1 / 2}}\right|,
$$

where $\mathcal{E}_{i}$ and $\mathcal{E}_{j}$ are subsequent pulse envelopes at delays $t_{1}-t_{2}$ typically chosen to integer multiples of the inverse repetition rate of the pulse train. Angle brackets denote ensemble averaging over independent SC pulses. When all pulses in the ensemble are identical, $g_{12}^{(1)} \equiv 0$, and all pulses in train can be compressed to identical short duration. For applications in precision frequency metrology, attosecond science, and high-field physics, however, a spectral coherence definition is usually more appropriate as all these applications rely on coherence between individual modes. The latter coherence is usually referred to as intrapulse coherence [29]:

$$
\Gamma_{\mathrm{CEP}}(\lambda)=\frac{\left|\left\langle\widetilde{\mathcal{E}}_{i}^{2}(2 \lambda) \widetilde{\mathcal{E}}_{i}^{*}(\lambda)\right\rangle\right|}{\left\langle\left|\widetilde{\mathcal{E}}_{i}^{2}(2 \lambda) \widetilde{\mathcal{E}}_{i}^{*}(\lambda)\right|\right\rangle} .
$$

In particular, the form of Eq. (14) is applicable to $f-2 f$ interferometry for carrier-envelope phase (CEP) measurement and stabilization $[27,64]$. Here $\Gamma_{\mathrm{CEP}} \equiv 1$ means that two spectral components are coherent, which provides perfect fringe contrast in an $f-2 f$ interferometer. In contrast, when $\Gamma_{\mathrm{CEP}}$ approaches zero, the fringe contrast in the $f-2 f$ interferometer is decreased, which hampers sensible stabilization. It is important to understand that both coherence definitions are not directly correlated; i.e., there can be near-perfect interpulse coherence without intrapulse coherence and vice versa.

Our previous study revealed an influence of the exact dispersion profile on spectral coherence [59], emulating a photonic-crystal fiber scenario. As in the latter, the source of decoherence effects is actually the buildup of ASE in the driver laser. Here we adapt this commonly used model to the design of a MPC. In order to simulate quantum noise in a realistic manner, we adapt the model from Refs. [59,65], which also includes noise accumulation within the driver laser cavity, limiting pulse contrast. To this end, the intracavity complex field after the $k$ th roundtrip in the laser is modeled as a coherent pulse and an additional incoherent ASE noise contribution. Here we define $\mathcal{E}_{\mathrm{t}}=\mathcal{E}_{\mathrm{c}}+\mathcal{E}_{k}^{\mathrm{ASE}}$, where $\mathcal{E}_{\mathrm{t}}, \mathcal{E}_{\mathrm{c}}$, and $\mathcal{E}_{k}^{\mathrm{ASE}}$ are the total field, coherent field, and ASE-induced incoherent field contribution, respectively. Considering the buildup process of ASE noise, which grows from random photons, $\mathcal{E}_{k}^{\text {ASE }}$ gradually grows with round trip. Moreover, an effective saturable absorber mechanism eventually limits the growth of the ASE according to

$$
\begin{aligned}
& \mathcal{E}_{1}^{\mathrm{ASE}}=\mathcal{E}_{1}^{\mathrm{RAN}}, \\
& \mathcal{E}_{k}^{\mathrm{ASE}}=\sqrt{1-\alpha} \mathcal{E}_{k-1}^{\mathrm{ASE}}+\mathcal{E}_{k}^{\mathrm{RAN}} .
\end{aligned}
$$

Here $\alpha$ is the modulation depth of the saturable absorber, and $\mathcal{E}_{k}^{\text {RAN }}$ denotes the complex amplitude random photons that is added to ASE noise per round trip. The random field modeled by

$$
\mathcal{E}_{k}^{\mathrm{RAN}}=\sqrt{P^{\mathrm{RAN}}} \exp \left(i \omega_{k} t+i \phi_{k}\right),
$$

where $P^{\mathrm{RAN}}=\gamma P_{\text {in }}$ is the effective peak power of the random field, $\gamma$ is the ratio between peak power of random and coherent field, and $\omega_{k}$ and $\phi_{k}$ are stochastic variables. In order to obtain reasonable estimates for $\gamma$, the intensity contrast of a mode-locked laser, i.e., $\chi$, is introduced, which can be written as $\chi=\alpha / \gamma^{2}$ [65]. The value of $\chi$ can be measured experimentally. For example, $\chi \approx 10^{7}$ has been observed for a Kerr-lens mode-locked Ti:sapphire laser [66]. Subsequently, we choose three different $\chi$ values, namely, $10^{6}, 10^{7}$, and $10^{8}$ for comparison.

Simulation results are shown in Fig. 8. Here 50 random inputs $\mathcal{E}_{k}^{\mathrm{ASE}}$ were employed as an ensemble for each value of $\chi$. The individually generated SCs and their spread are represented by the underlying gray curves in Fig. 8, and the ensemble averages are shown on top of these as red, blue, and pink curves to represent the 1-, 5-, and 10-mm cases, respectively. For low ASE contrast values $\left(\chi=10^{6}\right)$, intensity fluctuations are clearly discernible in the spectral wings whereas they nearly disappear at higher contrast levels $\left(\chi=10^{8}\right)$. All spectra are truncated at the $-60 \mathrm{~dB}$ level. The previously discussed blue shift for short lengths $d$ of silica is clearly visible in this representation at the low-wavelength cutoff near $600 \mathrm{~nm}$. This trend affects the interpulse coherence $g_{12}^{(1)}$ in Figs. $8(\mathrm{~b}), 8(\mathrm{e})$, and $8(\mathrm{~h})$ as well; i.e., the compressible part of the spectrum is also blue shifted. Apart from this spectral shift, however, at fixed contrast, the width of the coherent part of the spectrum $\left(g_{12}^{(1)}>0.9\right)$ remains mostly unaffected when measured in optical octaves. Coherence in the $\chi=10^{6}$ spectra encompasses $\approx 1.2$ octaves, which increases to $>1.5$ octaves with high ASE contrast input pulses; cf. Figs. 8(e) 

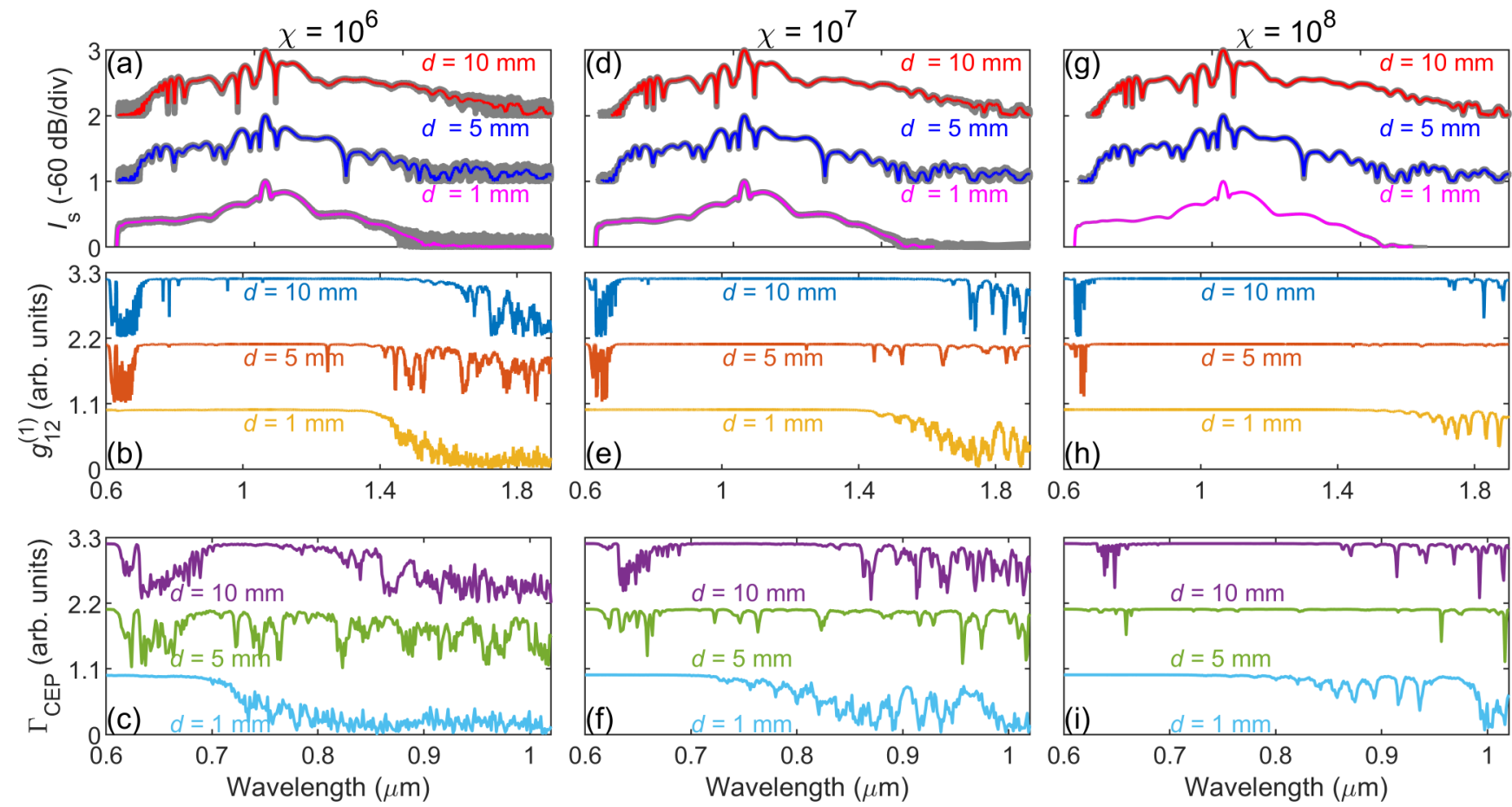

FIG. 8. Coherence properties of the supercontinua as a function of input pulse contrast (columns), with $\chi=10^{6}, 10^{7}$, and $10^{8}$ from left to right. [(a), (d), (g)] Top row depicts the spectral intensity of the SC and their fluctuations (gray background). [(b), (e), (h)] Middle column: interpulse coherence $g_{12}^{(1)}$. [(c), (f), (i)] Bottom column: intrapulse coherence $\Gamma_{\mathrm{CEP}}$. Different curve colors refer to cases $d=10,5$, and $1 \mathrm{~mm}$ as indicated by labeling in the individual subfigure. $d=5 \mathrm{~mm}$ and $d=10 \mathrm{~mm}$ traces have been offset by one and two ordinate units for clarity, respectively.

and $8(\mathrm{~h})$. Therefore, all these spectra appear perfectly compressible, at least at the $-20 \mathrm{~dB}$ level, as noise amplification induced by modulation instability can be suitably avoided [23] in the normal dispersion regime.

Intrapulse coherence is depicted in Figs. 8(c), 8(f) and 8(i), where the wavelength scale has been chosen for the $f$ component. In order to exploit the SC for CEP stabilization, one has to heterodyne a frequency-doubled $2 f$ component from the infrared edge of the spectrum with an $f$ component near the blue cutoff. This different type of coherence exhibits a similar influence of the blue shift, yet a much higher sensitivity to ASE contrast. For $\chi=10^{6}$, high intrapulse coherence can only be observed in a rather narrow 100-nm interval, which increases to $400 \mathrm{~nm}$ at $\chi=10^{8}$. There is no clear trend concerning the dependence on $d$. The best overall intrapulse coherence is observed for $d=10 \mathrm{~mm}$ and $\chi=10^{8}$; the worst at identical $d$ and $\chi=10^{8}$; cf. Figs. 8(c) and 8(i). This conclusion can be nearly identically made for the interpulse coherence. Meaningful application of multipass supercontinua therefore heavily relies on the ASE contrast of the laser source and is less susceptible to the exact design of the multipass cell.

To shed more light on CEP-sensitive applications, we extracted the spectral phase difference between the two spectral components at $\lambda$ and $2 \lambda$ from Eq. (14) using [29]

$$
\Delta \phi_{i}=\arg \left[\widetilde{\mathcal{E}}_{i}^{2}(2 \lambda) \widetilde{\mathcal{E}}_{i}^{*}(\lambda)\right]=2 \phi_{i}^{(2 \lambda)}-\phi_{i}^{(\lambda)},
$$

where $\phi_{i}^{2 \lambda}$ and $\phi_{i}^{\lambda}$ are the spectral phases of $\widetilde{\mathcal{E}}_{i}$ at $2 \lambda$ and $\lambda$, respectively. After ensemble averaging over Eq. (17), one yields a constant phase difference of $\left\langle\phi_{i}\right\rangle$. Phase fluctuations amount to

$$
\sigma_{\phi}=\sqrt{\left\langle\left(\Delta \phi_{i}-\left\langle\Delta \phi_{i}\right\rangle\right)^{2}\right\rangle},
$$

where $\sigma_{\phi}$ essentially measures the fidelity of coherent phase transfer in the process of SC generation. Therefore, when $\sigma_{\phi} \equiv 0$, the CEP can be tightly locked. In order to understand the variation of this fidelity for various values of thickness and intensity contrast, Eqs. (17) and (18) are numerically solved. The dependence of $\sigma_{\phi}$ on wavelength is shown in Fig. 9. This consideration essentially confirms the trends discussed above and leads to similar wavelength ranges that can be used to establish CEP stabilization with low residual phase jitters $(<100 \mathrm{mrad})$. For $\chi=10^{6}$, this range encompasses $100 \mathrm{~nm}$ [Fig. 9(a)] and reaches $\geqslant 300 \mathrm{~nm}$ for $\chi=10^{8}$.

\section{CONCLUSIONS}

Our numerical simulations indicate a pulse collapse scenario at large values of $d$, which is mitigated in the quasicontinuous case of $d=1 \mathrm{~mm}$. Similar collapse mechanisms have been previously observed in filament compression [17]. As this collapse was often accompanied by large spatial inhomogeneities, this issue degraded the utility of filament compression. In contrast, in the multipass cell approach, spatial inhomogeneities are widely suppressed by the filtering action of the cavity. This filtering action becomes more effective with a large number of cavity passes; i.e., the quasicontinuous case of short length $d$ is most favorable here. This case is accompanied by a pronounced blue shift of the supercontinuum. A similar blue shift also appears in filament 

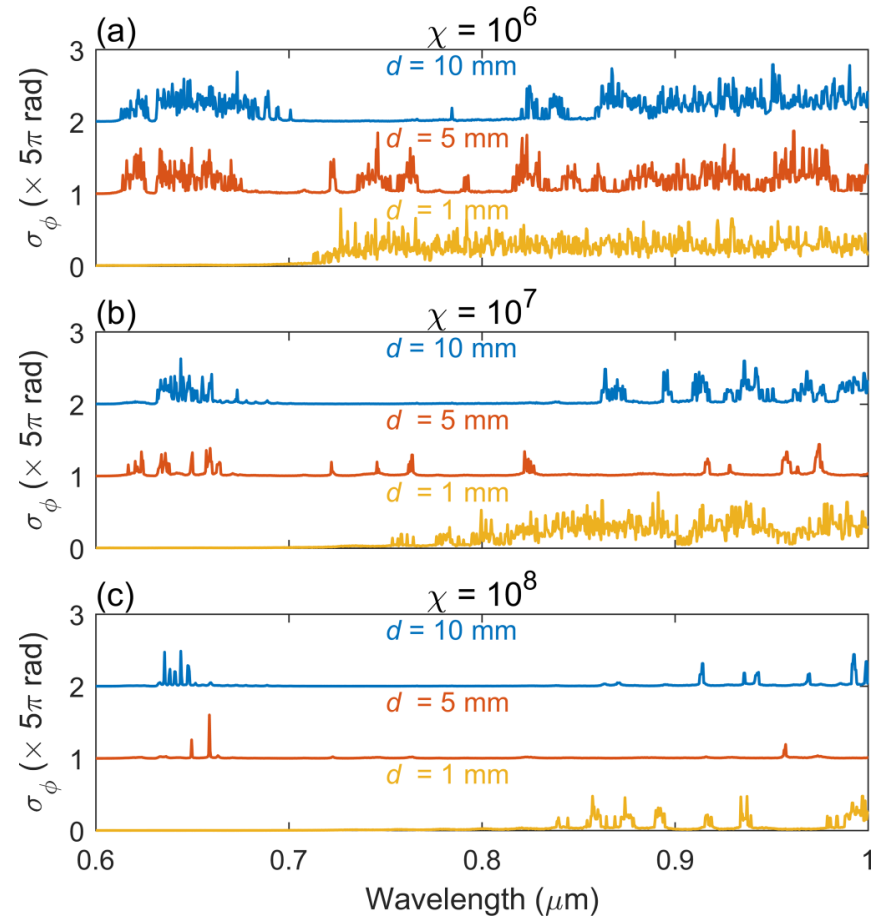

FIG. 9. Residual phase jitters $\sigma_{\phi}$ vs wavelength of the $f$ component in simulated CEP stabilization for (a) $\chi=10^{6}$, (b) $\chi=10^{7}$, and (c) $\chi=10^{8}$. Different traces refer to different values of $d$ as indicated in the subfigures. Traces for $d=5 \mathrm{~mm}$ and $d=10 \mathrm{~mm}$ are offset by one and ordinate units for clarity, respectively.

compression and has often been related to plasma effects. At the intensity levels considered in this work, one can safely exclude such effects. We therefore find space-time focusing effects [62] as the only reasonable explanation for the blue shift. Compared to filament compression, multipass cells can therefore exploit the additional spectral broadening due to the blue shifting without suffering from detrimental plasma losses, pulse breakups, or spatial inhomogeneities.
In our simulations, the $d=1 \mathrm{~mm}$ case stands out as it provides shortest pulses together with the highest compression efficiency and best spatial homogeneity. Assuming 100-fs input pulses, we observe an $\approx 15$-fold compression to $6.6 \mathrm{fs}$. Given that the compression takes place in a material with normal dispersion below $1.3 \mu \mathrm{m}$, one does not expect any rapid degradation of interpulse coherence or compressibility due to input energy fluctuations. These expectations are widely confirmed in our numerical simulations. However, these beneficial temporal coherence properties do not automatically allow us to conclude similarly favorable spectral coherence properties as they are important for CEP stabilization schemes. CEP stabilization relies on spectral coherence of spectral components one octave apart. In particular, when octave coverage is barely reached, the power levels of these components in the spectral wings are heavily affected by pulse energy fluctuations and input ASE noise. As our simulations indicate, we do not see any clear trend between the intrapulse coherence and multipass cavity design. Instead, we see a rather strong influence on the ASE contrast of the input pulses. It therefore requires pulses of excellent pulse contrast $\chi \geqslant 10^{8}$ to avoid detrimental effects in CEP stabilization schemes. Nevertheless, spectral coherence properties and compressibility are orthogonal aspects, which can be individually optimized without overly large interplay. Compared to other compression schemes, multipass cells display a larger range of possibilities for optimization and engineering. We therefore believe that our simulations will enable the design of multipass compression schemes with superior properties, which go significantly beyond the capabilities of current designs.

\section{ACKNOWLEDGMENTS}

C. Mei thanks Dr. T. M. Kardaś for helpful discussions. This work was supported by the Sino-German (CSC-DAAD) Postdoc Scholarship Program, 2019 (No. 57460082).
[1] E. B. Treacy, Optical pulse compression with diffraction gratings, IEEE J. Quantum Electron. 5, 454 (1969).

[2] W. J. Tomlinson, R. H. Stolen, and C. V. Shank, Compression of optical pulses chirped by self-phase modulation, J. Opt. Soc. Am. B 1, 139 (1984).

[3] E. M. Dianov, A. Y. Karasik, P. V. Mamyshev, G. I. Onishchukov, A. M. Prokhorov, M. F. Stel'makh, and A. A. Fomichev, 100-fold compression of picosecond pulses from a parametric light source in single-mode optical fibers at wavelengths 1.5-1.65 $\mu \mathrm{m}$, JETP Lett. 40, 903 (1985).

[4] E. M. Dianov, A. Karasik, P. Mamyshev, A. Prokhorov, and D. Fursa, Production of high-contrast subpicosecond pulses by a single-stage 110 -fold compression of YAG- $\mathrm{Nd}^{3+}$ laser-pulses, Kvantovata Elektronika 14, 662 (1987).

[5] E. M. Dianov, L. Ivanov, P. Mamyshev, and A. Prokhorov, Efficient compression of high-energy pulses, IEEE J. Quantum Electron. 25, 828 (1989).
[6] W. J. Tomlinson and W. H. Knox, Limits of fiber-grating optical pulse compression, J. Opt. Soc. Am. B 4, 1404 (1987).

[7] K. Smith and L. F. Mollenauer, Experimental observation of adiabatic compression and expansion of soliton pulses over long fiber paths, Opt. Lett. 14, 751 (1989).

[8] T. Nagy, S. Hädrich, P. Simon, A. Blumenstein, N. Walther, R. Klas, J. Buldt, H. Stark, S. Breitkopf, P. Jójárt, I. Seres, Z. Várallyay, T. Eidam, and J. Limpert, Generation of three-cycle multi-millijoule laser pulses at $318 \mathrm{~W}$ average power, Optica 6 , 1423 (2019).

[9] T. Nagy, M. Kretschmar, M. J. J. Vrakking, and A. Rouzée, Generation of above-terawatt 1.5 -cycle visible pulses at $1 \mathrm{kHz}$, Opt. Lett. 45, 3313 (2020).

[10] M. Nisoli, S. De Silvestri, and O. Svelto, Generation of high energy 10 fs pulses by a new pulse compression technique, Appl. Phys. Lett. 68, 2793 (1996). 
[11] M. Nisoli, S. De Silvestri, O. Svelto, R. Szipöcs, K. Ferencz, C. Spielmann, S. Sartania, and F. Krausz, Compression of highenergy laser pulses below 5 fs, Opt. Lett. 22, 522 (1997).

[12] B. Schenkel, J. Biegert, U. Keller, C. Vozzi, M. Nisoli, G. Sansone, S. Stagira, S. De Silvestri, and O. Svelto, Generation of 3.8-fs pulses from adaptive compression of a cascaded hollow fiber supercontinuum, Opt. Lett. 28, 1987 (2003).

[13] G. Steinmeyer and G. Stibenz, Generation of sub-4-fs pulses via compression of a white-light continuum using only chirped mirrors, Appl. Phys. B 82, 175 (2006).

[14] C. P. Hauri, W. Kornelis, F. W. Helbing, A. Heinrich, A. Couairon, A. Mysyrowicz, J. Biegert, and U. Keller, Generation of intense, carrier-envelope phase-locked few-cycle laser pulses through filamentation, Appl. Phys. B 79, 673 (2004).

[15] G. Stibenz, N. Zhavoronkov, and G. Steinmeyer, Selfcompression of millijoule pulses to $7.8 \mathrm{fs}$ duration in a white-light filament, Opt. Lett. 31, 274 (2006).

[16] S. Skupin, G. Stibenz, L. Bergé, F. Lederer, T. Sokollik, M. Schnürer, N. Zhavoronkov, and G. Steinmeyer, Selfcompression by femtosecond pulse filamentation: Experiments versus numerical simulations, Phys. Rev. E 74, 056604 (2006).

[17] C. Brée, A. Demircan, S. Skupin, L. Bergé, and G. Steinmeyer, Self-pinching of pulsed laser beams during filamentary propagation, Opt. Expr. 17, 16429 (2009).

[18] D. R. Herriott, H. Kogelnik, and R. Kompfner, Off-axis paths in spherical mirror interferometers, Appl. Opt. 3, 523 (1964).

[19] D. R. Herriott and H. J. Schulte, Folded optical delay lines, Appl. Opt. 4, 883 (1965).

[20] N. Milosevic, G. Tempea, and T. Brabec, Optical pulse compression: Bulk media versus hollow waveguides, Opt. Lett. 25, 672 (2000).

[21] M. Kaumanns, D. Kormin, T. Nubbemeyer, V. Pervak, and S. Karsch, Spectral broadening of $112 \mathrm{~mJ}, 1.3$ ps pulses at $5 \mathrm{kHz}$ in a donut mode multipass with compressibility to $37 \mathrm{fs}$, Opt. Lett. 46, 929 (2021).

[22] B. Schenkel, R. Paschotta, and U. Keller, Pulse compression with supercontinuum generation in microstructure fibers, J. Opt. Soc. Am. B 22, 687 (2005).

[23] J. M. Dudley, G. Genty, and S. Coen, Supercontinuum generation in photonic crystal fiber, Rev. Mod. Phys. 78, 1135 (2006).

[24] J. Herrmann, U. Griebner, N. Zhavoronkov, A. Husakou, D. Nickel, J. C. Knight, W. J. Wadsworth, P. St. J. Russell, and G. Korn, Experimental Evidence for Supercontinuum Generation by Fission of Higher-Order Solitons in Photonic Fibers, Phys. Rev. Lett. 88, 173901 (2002).

[25] T. W. Hänsch, Nobel lecture: Passion for precision, Rev. Mod. Phys. 78, 1297 (2006).

[26] T. Fortier and E. Baumann, 20 years of developments in optical frequency comb technology and applications, Commun. Phys. 2, 153 (2019).

[27] H. R. Telle, G. Steinmeyer, A. E. Dunlop, J. Stenger, D. H. Sutter, and U. Keller, Carrier-envelope offset phase control: A novel concept for absolute optical frequency measurement and ultrashort pulse generation, Appl. Phys. B 69, 327 (1999).

[28] A. Baltuška, T. Udem, M. Uiberacker, M. Hentschel, E. Goulielmakis, C. Gohle, R. Holzwarth, V. S. Yakovlev, A. Scrinzi, T. W. Hänsch, and F. Krausz, Attosecond control of electronic processes by intense light fields, Nature (London) 421, 611 (2003).
[29] N. Raabe, T. Feng, T. Witting, A. Demircan, C. Brée, and G. Steinmeyer, Role of Intrapulse Coherence in Carrier-Envelope Phase Stabilization, Phys. Rev. Lett. 119, 123901 (2017).

[30] S. N. Vlasov, E. V. Koposova, and V. E. Yashin, Spectral broadening and compression of highintensity laser pulses in quasi-periodic systems with Kerr nonlinearity, Quantum Electron. 42, 989 (2012).

[31] J. Schulte, T. Sartorius, J. Weitenberg, A. Vernaleken, and P. Russbueldt, Nonlinear pulse compression in a multi-pass cell, Opt. Lett. 41, 4511 (2016).

[32] M. Seidel, G. Arisholm, J. Brons, V. Pervak, and O. Pronin, All solid-state spectral broadening: An average and peak power scalable method for compression of ultrashort pulses, Opt. Express 24, 9412 (2016).

[33] J. Weitenberg, T. Saule, J. Schulte, and P. Rußbüldt, Nonlinear pulse compression to sub- $40 \mathrm{fs}$ at $4.5 \mu \mathrm{J}$ pulse energy by multipass-cell spectral broadening, IEEE J. Quantum Electron. 53, 8600204 (2017).

[34] J. Weitenberg, A. Vernaleken, J. Schulte, A. Ozawa, T. Sartorius, V. Pervak, H.-D. Hoffmann, T. Udem, P. Russbüldt, and T. W. Hänsch, Multi-pass-cell-based nonlinear pulse compression to $115 \mathrm{fs}$ at $7.5 \mu \mathrm{J}$ pulse energy and $300 \mathrm{~W}$ average power, Opt. Expr. 25, 20502 (2017).

[35] E. Vicetini, Y. Wang, D. Gatti, A. Gambetta, P. Laporta, G. Ganzerano, K. Curtis, K. Mcewan, C. R. Howle, and N. Coluccellio, Nonlinear pulse compression to 22 fs at $15.6 \mu \mathrm{J}$ by an all-solid-state multipass approach, Opt. Expr. 28, 4541 (2020).

[36] G. Jargot, N. Daher, L. Lavenu, X. Delen, N. Forget, M. Hanna, and P. Georges, Self-compression in a multipass cell, Opt. Lett. 43, 5643 (2018).

[37] M. Kaumanns, V. Pervak, D. Kormin, V. Leshchenko, A. Kessel, M. Ueffing, Y. Chen, and T. Nubbemeyer, Multipass spectral broadening of $18 \mathrm{~mJ}$ pulses compressible from $1.3 \mathrm{ps}$ to $41 \mathrm{fs}$, Opt. Lett. 43, 5877 (2018).

[38] L. Lavenu, M. Nattle, F. Guichard, Y. Zaouter, X. Delen, M. Hanna, E. Mottay, and P. Georges, Nonlinear pulse compression based on a gas-filled multipass cell, Opt. Lett. 43, 2252 (2018).

[39] M. Ueffing, S. Reiger, M. Kaumanns, V. Pervak, M. Trubetskov, T. Nubbemeyer, and F. Krausz, Nonlinear pulse compression based on a gas-filled multipass cell, Opt. Lett. 43, 2070 (2018).

[40] P. Russbueldt, J. Weitenberg, J. Schulte, R. Meyer, C. Meinhardt, H. D. Hoffmann, and R. Poprawe, Scalable 30 fs laser source with $530 \mathrm{~W}$ average power, Opt. Lett. 44, 5222 (2019).

[41] H. Cao, R. S. Nagymihaly, and M. Kalashnikov, Relativistic near-single-cycle optical vortex pulses from noble gas-filled multipass cells, Opt. Lett. 45, 3240 (2020).

[42] S. Gröbmeyer, K. Fritsch, B. Schneider, M. Poetzlberger, V. Pervak, J. Brons, and O. Pronin, Self-compression at $1 \mu \mathrm{m}$ wavelength in all-bulk multi-pass geometry, Appl. Phys. B 126, 159 (2020).

[43] K. Fritsch, M. Poetzlberger, V. Pervak, J. Brons, and O. Pronin, All-solid-state multipass spectral broadening to sub-20 fs, Opt. Lett. 43, 4643 (2018).

[44] G. Steinmeyer, Dispersion oscillations in ultrafast phasecorrection devices, IEEE J. Quantum Electron. 39, 1027 (2003).

[45] A. Diebold, F. Saltarelli, I. J. Graumann, C. J. Saraceno, C. R. Phillips, and U. Keller, Gas-lens effect in kW-class thin-disk lasers, Opt. Expr. 26, 12648 (2018). 
[46] M. Hanna, X. Délen, L. Lavenu, F. Guichard, Y. Zaouter, F. Druon, and P. Georges, Nonlinear temporal compression in multipass cells: Theory, J. Opt. Soc. Am. B 34, 1340 (2017).

[47] M. Kolesik, J. V. Moloney, and M. Mlejnek, Unidirectional Optical Pulse Propagation Equation, Phys. Rev. Lett. 89, 283902 (2002).

[48] T. M. Kardaś, Y. Stepanenko, and C. Radzewicz, Noncollinear and nonlinear pulse propagation, Sci. Rep. 8, 14350 (2018).

[49] T. E. Lanier and J. R. Gulley, Nonlinear space-time focusing and filamentation of annular femtosecond pulses in dielectrics, J. Opt. Soc. Am. B 33, 292 (2016).

[50] M. A. Eftekhar, Z. Sanjabi-Eznaveh, H. E. Lopez-Aviles, S. Benis, J. E. Antonio-Lopez, M. Kolesik, F. Wise, R. AmezcuaCorrea, and D. N. Christodoulides, Accelerated nonlinear interactions in graded-index multimode fibers, Nat. Commun. 10, 1638 (2019).

[51] A. Couairon, E. Brambilla, T. Corti, D. Majus, O. de J. Ramírez Góngora, and M. Kolesik, Practitioner's guide to laser pulse propagation models and simulation, Eur. Phys. J. Spec. Top. 199, 5 (2011).

[52] L. Bergé, S. Skupin, R. Nuter, J. Kasparian, and J.-P. Wolf, Ultrashort filaments of light in weakly ionized, optically transparent media, Rep. Prog. Phys. 70, 1633 (2007).

[53] L. V. Keldysh, Ionization in the field of a strong electromagnetic wave, Sov. Phys. JETP 20, 1307 (1965).

[54] S. Tzortzakis, L. Sudrie, M. Franco, B. Prade, A. Mysyrowicz, A. Couairon, and L. Bergé, Self-Guided Propagation of Ultrashort IR Laser Pulses in Fused Silica, Phys. Rev. Lett. 87, 213902 (2001).

[55] D. M. Rayner, A. Naumov, and P. B. Corkum, Ultrashort pulse non-linear optical absorption in transparent media, Opt. Expr. 13, 3208 (2005).
[56] J. H. Marburger, Self-focusing: Theory, Progr. Quantum Electron. 4, 35 (1975).

[57] L. Yu, M. Huang, and M. Chen, Quasi-discrete Hankel transform, Opt. Lett. 23, 409 (1998).

[58] P. Kabacinski, T. M. Kardas, Y. Stepanenko, and C. Radzewicz, Nonlinear refractive index measurement by SPM-induced phase regression, Opt. Expr. 27, 11018 (2019).

[59] C. Mei and G. Steinmeyer, Tailoring the waveguide dispersion of nonlinear fibers for supercontinuum generation with superior intrapulse coherence, J. Opt. Soc. Am. B 37, 2485 (2020).

[60] S. Skupin and L. Bergé, Self-guiding of femtosecond light pulses in condensed media: Plasma generation versus chromatic dispersion, Physica D (Amsterdam, Neth.) 220, 14 (2006).

[61] A. Brodeur and S. L. Chin, Ultrafast white-light continuum generation and self-focusing in transparent condensed media, J. Opt. Soc. Am. B 16, 637 (1999).

[62] A. L. Gaeta, Catastrophic Collapse of Ultrashort Pulses, Phys. Rev. Lett. 84, 3582 (2000).

[63] J. M. Dudley and S. Coen, Coherence properties of supercontinuum spectra generated in photonic crystal and tapered optical fibers, Opt. Lett. 27, 1180 (2002).

[64] M. Natile, A. Golinelli, L. Lavenu, F. Guichard, M. Hanna, Y. Zaouter, R. Chiche, X. Chen, J. F. Hergott, W. Boutu, H. Merdji, and P. Georges, CEP-stable high-energy ytterbium-doped fiber amplifier, Opt. Lett. 44, 3909 (2019).

[65] R. Liao, C. Mei, Y. Song, A. Demircan, and G. Steinmeyer, Spontaneous emission noise in mode-locked lasers and frequency combs, Phys. Rev. A 102, 013506 (2020).

[66] A. Braun, J. V. Rudd, H. Cheng, G. Mourou, D. Kopf, I. D. Jung, K. J. Weingarten, and U. Keller, Characterization of short-pulse oscillators by means of a high-dynamic-range autocorrelation measurement, Opt. Lett. 20, 1889 (1995). 